\title{
Profile of pacritinib and its potential in the treatment of hematologic disorders
}

This article was published in the following Dove Press journal:

Journal of Blood Medicine

19 August 2014

Number of times this article has been viewed

\section{Eleftheria Hatzimichael \\ Evangelos Tsolas \\ Evangelos Briasoulis}

Department of Haematology, University Hospital of Ioannina, loannina, Greece
Correspondence: Eleftheria Hatzimichael Department of Haematology, University Hospital of loannina, St Niarchou Ave, 45500, loannina, Greece

Tel +302651099615

Email ehatzim@cc.uoi.gr
Abstract: Pacritinib (previously known as SB-1518) is an innovative selective inhibitor of Janus kinase 2 and FMS-related tyrosine kinase 3 providing potential in the treatment of hematological malignancies such as myeloproliferative neoplasias, acute myeloid leukemia, and various lymphomas. Pacritinib has potent antiproliferative activity in Janus kinase 2 and/or FMS-related tyrosine kinase 3 activity-dependent cell lines and an ability to promote apoptosis and inhibit the signal transducers and activators of transcription (STAT) pathway. Pharmacokinetic studies have indicated a good per os bioavailability and favorable kinetic parameters. To date, promising results have been produced in five completed early-phase clinical trials in which pacritinib has been studied. Pacritinib displayed interesting activity and an acceptable safety profile, with mild to moderate gastrointestinal disorders being its most common adverse effects.

Keywords: pacritinib, SB-1518, JAK inhibitors, myeloproliferative neoplasms, acute myeloid leukemia, lymphoma, treatment

\section{Introduction}

Pacritinib (previously known as SB-1518) is an innovative selective inhibitor of Janus kinase 2 (JAK2) and FMS-related tyrosine kinase 3 (FLT3) providing potential in the treatment of hematologic malignancies such as myeloproliferative neoplasias (MPNs), acute myeloid leukemia (AML), and various lymphomas. Pacritinib has potent antiproliferative activity in JAK2 and/or FLT3 activity-dependent cell lines and the ability to promote apoptosis and inhibit the signal transducers and activators of transcription (STAT) pathway. In this review, we profile pacritinib and summarize its clinical development and give an overview of the pathways it is involved in and the diseases in which it has already been tested.

\section{Dysregulation in the JAK-STAT pathway in hematological malignancies}

The JAK family consists of four nonreceptor intracellular tyrosine kinases (JAK1, JAK2, JAK3, and tyrosine kinase 2 [TYK2]) that are closely associated with cytokine receptors and that transduce signals to the nucleus on activation. Although in humans JAK 3 is predominately expressed in hematopoietic cells and colon endothelial cells, the remaining JAK family members, JAK1, JAK 2, and TYK2, are ubiquitously expressed. The engagement of a cytokine or growth factor to one of their transmembrane cytokine receptors leads to transphosphorylation of the JAK family members that are associated with them and the recruitment and phosphorylation of STAT proteins on tyrosine residues. ${ }^{1}$ The phosphorylated STATs (pSTATs) are then dimerized 
and enter the nucleus, where they regulate the expression of a wide spectrum of genes involved in biological processes, such as cell proliferation, angiogenesis, immune response, and apoptosis (Figure 1). ${ }^{2}$

A specific mutation in the tyrosine-protein kinase JAK2, JAK2V617F, is present in the majority of patients with MPNs (almost all patients with polycythemia vera [PV] and half of the patients with primary myelofibrosis [PMF] and essential thrombocythemia [ET]), which leads to an overactivation of the JAK2-STAT pathway (gain-of-function mutation) by reducing the hindering effect of the pseudokinase on the kinase portion of the molecule..$^{3,4}$ The presence of this mutation has been described in other hematological malignancies as well, but to a much lesser extent: roughly in 3\%-5\% of patients with myelodysplastic syndromes and less than $5 \%$ of patients with AML. ${ }^{5,6}$

Subsequent studies identified $J A K 2$ exon 12 mutation in patients with JAK2 V617F-negative PV and mutations in the $M P L$ gene, encoding for the thrombopoietin receptor, in patients with JAK2 V617F-negative ET and PMF. Most recently, another mutation was identified in patients with JAK2 V617F-negative and MPL-negative MPNs in the gene encoding calreticulin $(C A L R))^{7,8}$ In all cases, the presence of the above-mentioned hallmark mutations leads to an activated JAK-STAT signaling, suggesting this pathway is critical for MPN pathogenesis.

JAK2 gain-of-function mutations are rarely observed in lymphomas. However, aberrant expression of proteins involved in the JAK-STAT pathway, leading to its activation, has been described in a variety of lymphomas such as diffuse large B-cell lymphomas (DLBCL), Hodgkin's lymphomas (HL), and primary mediastinal B-cell lymphoma, ${ }^{9,10}$ with a dual role of promoting cell proliferation and immune evasion, making it an appealing target for pathway-directed therapy. JAK2 amplifications and gains have been described in up to a third of patients with HL and $30 \%-50 \%$ of patients with primary mediastinal B-cell lymphoma, ${ }^{11,12}$ two different neoplasms that share several common features. For example, in nodular sclerosis HL with amplification of 9p24.1, it has been shown that not only programmed cell death -1 (PD-1) ligand genes residing in this genomic locus are overexpressed but also $J A K 2$ located $322 \mathrm{~kb}$ upstream from $P D-L 1$ on $9 \mathrm{p} 24.1$. $J A K 2$ amplification was also shown to increase and specifically induce PD-1 ligand transcription and to enhance sensitivity

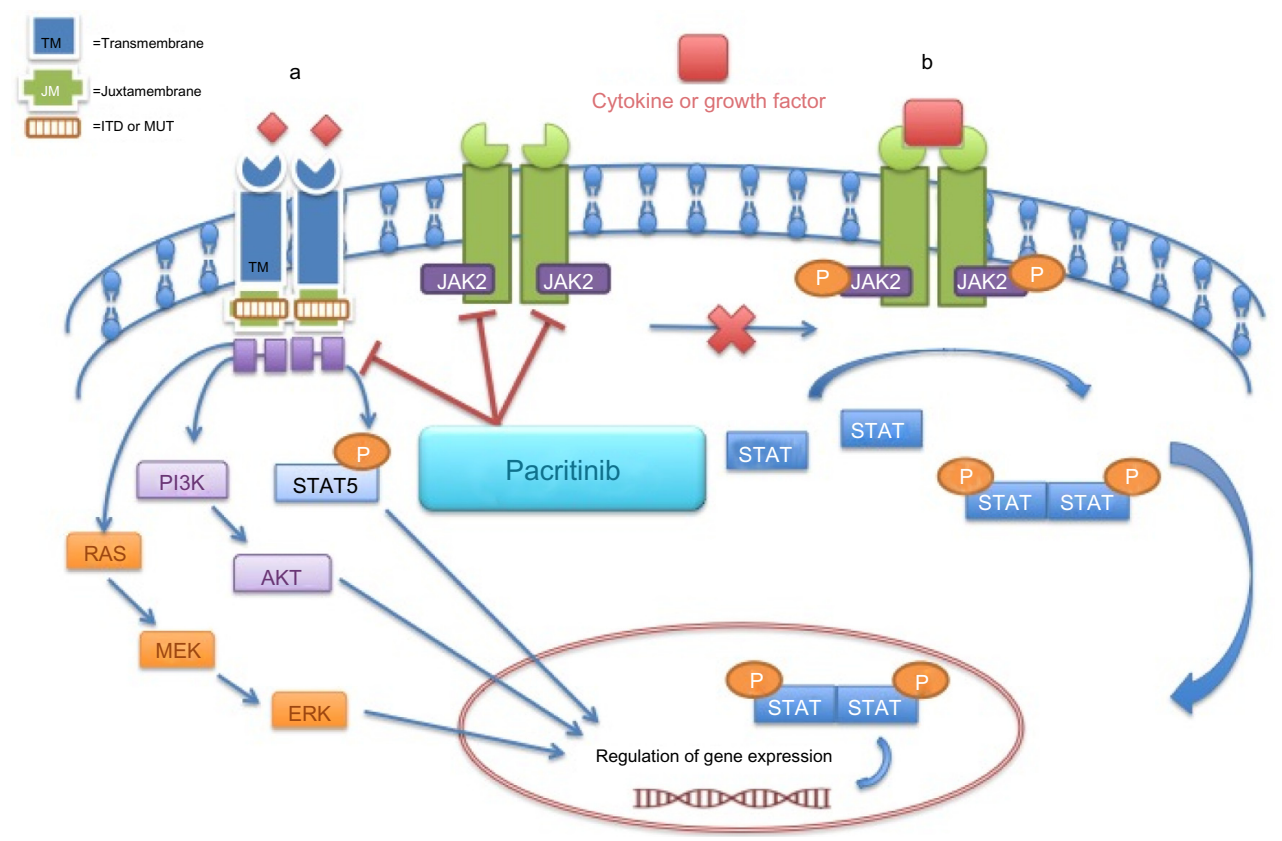

Figure I Inhibitory effects of pacritinib in the FMS-like tyrosine kinase (FLT3) and Janus kinase (JAK)—signal transducer and activator of transcription (STAT) pathway. Notes: (a) The FLT3 receptor is composed of five extracellularimmunoglobulin-like domains, a transmembrane domain (TM), a juxtamembrane domain (JM) and a tyrosinekinase domain consisting of 2 domains that are connected by a tyrosine-kinase insert. FLT3 with internal tandem duplication (ITD) or mutation (MUT) is activated independently of ligands at the cell surface. FLT3-ITD mutations, as well as TKD mutations, result in the constitutive activation of FLT3 kinase and its downstream proliferative signaling pathways, including the RAS/MAPK kinase (MEK)/extracellular signal-regulated kinase (ERK) pathway and PI3K/AKT pathway. In addition, and in contrast to wild-type FLT3 signaling, FLT3-ITD potently activates the STAT5 pathway inducing expression of genes that are important for cell growth. Pacritinb inhibits FLT3, FLT3-ITD and FLT3D835Y mutant blocking the downstream effects of this pathway. (b) The Janus kinase (JAK) - signal transducer and activator of transcription (STAT) pathway is also depicted. The JAK2 receptor is activated by a signal from cytokines or growth factors. Activation of JAK2 leads to phosphorylation of STAT proteins, allowing for dimerization of the STAT, which then migrate into the cytoplasm and translocate into the nucleus, allowing for transcription of their target genes. Pacritinib inhibits JAK2, both wild type and mutant forms blocking this pathway as well. 
to JAK2 inhibition, defining the PD-1 pathway and JAK2 as complementary rational therapeutic targets. Loss-of-function mutations in SOCS-1 that lead to delayed degradation of the phosphorylated JAK2 and increased activity of STAT $5^{13,14}$ and gene rearrangements by translocation involving the $J A K 2$, such as $\mathrm{t}(4 ; 9)(\mathrm{q} 21 ; \mathrm{p} 24)$, have also been described in primary mediastinal B-cell lymphoma and HL. ${ }^{15}$ Interestingly, activation of JAK-STAT signaling has been observed even in the absence of genetic abnormalities in lymphomas. An aberrant dysregulation of a network of cytokines in the microenvironment, such as interleukin $10,{ }^{16,17}$ has recently been implicated in the activation of the pathway.

JAK2V617 mutations are rare in AML patients and found more commonly in patients with $\mathrm{t}(8: 21) .{ }^{18,19}$ Increased levels of phosphorylated JAK2 were found to be a predictor of poor response to chemotherapy and a factor of poor prognosis, ${ }^{20}$ which justifies its consideration as a therapeutic target in this disease. ${ }^{21}$ Increased activity of STAT3 has been reported in $20 \%-50 \%$ of AML patients, although it is still unclear how this contributes to the pathogenesis of the disease. ${ }^{22}$

\section{FLT3 and hematological malignancies}

FMS-related tyrosine kinase 3 (FLT3) is a class 3 transmembrane tyrosine kinase receptor that stimulates cell proliferation on activation and is expressed in immature hematopoietic cells, placenta, brain, gonads, and lymphohematopoietic organs such as the liver, the spleen, and the thymus. ${ }^{23,24}$ It is also expressed in various hematologic malignancies including most AML subtypes, B-cell acute lymphoblastic leukemia (ALL) some T-cell ALL, and chronic myelogenous leukemia in blast crisis. ${ }^{21}$ FLT-3 receptor becomes activated when bound to its FLT3 ligand (FLT3L), which leads to its unfolding and homodimerization. The formation of homodimers switches on its intrinsic tyrosine kinase activity and recruits a number of intracellular proteins to its intracellular domain. The target molecules are phosphorylated and activated, and then they enter the cell nucleus, where they regulate the expression of many genes related to cell survival, proliferation, and differentiation. ${ }^{25}$ FLT3 mutations are either internal tandem duplications (ITD) of 300-400 bp or point mutations. FLT3-ITD results from a duplication of a fragment within the juxtamembrane domain-coding region. These abnormalities lead to overactivation of the FLT3 pathway and dysregulation of the physiological processes that it controls (Figure 1). Up to one third of patients with AML have one of these mutations, and their prognosis is very poor. ${ }^{26}$

\section{Myeloproliferative neoplasms}

MPNs include, among other entities, PMF, PV, and ET. Patients with PV and ET have a more protracted clinical course and are prone to vascular and thromboembolic events, whereas patients with PMF suffer from much more constitutional symptoms and have a more rapid disease progression and a higher predisposition to progress to AML. ${ }^{27} \mathrm{MF}$ may also develop from another MPN (postessential thrombocythemia MF [PET-MF] or postpolycythemia vera MF [PPV-MF]).

\section{JAK inhibitors in MF}

The only curative treatment for PMF is allogeneic stem cell transplantation. Candidates for allogeneic stem cell transplantation are traditionally young patients with two or more adverse features (ie, hemoglobin levels $<10 \mathrm{~g} / \mathrm{dL}$, isolated cytogenetic abnormality, constitutional symptoms, or blasts lower than 1\%). ${ }^{28}$ However, not many patients undergo allogeneic stem cell transplantation because of advanced age, presence of comorbidities, or lack of donor; therefore, treatment is either supportive (eg, transfusions) or palliative, aiming for control (eg, hydroxyurea and splenic irradiation). Androgens have also been shown to improve anemia in patients who are not transplant candidates, ${ }^{29}$ whereas erythropoietin and immunomodulatory agents with or without steroids have also been used with satisfactory results; however, their use is still off-label..$^{30} \mathrm{~A}$ major breakthrough in the delineation of the pathogenesis of MPNs was the identification of the JAK2V617F mutation, which ultimately led to the development of JAK2 inhibitors. At this time, more than 10 JAK kinase inhibitors have been advanced into clinical trials, and one of them, ruxolitinib, was recently approved for patients with MF, either primary or postpolycythemia or postthrombocythemia. Controlled Myelofibrosis Study with Oral JAK Inhibitor Treatment I and Controlled Myelofibrosis Study with Oral JAK Inhibitor Treatment II are the clinical trials on which this approval was based. They demonstrated that splenomegaly was reduced and constitutional symptoms were improved, but hematological toxicity was notable (anemia and thrombocytopenia). ${ }^{31}$

\section{AML}

AML is classified, using the World Health Organization classification system on a combination of morphology, immunophenotype, genetic, and molecular abnormalities and clinical features. This classification attempts to identify biologic entities that are amenable to targeted therapy, or at least to a treatment plan that will provide a better overall survival with fewer relapses. Certain mutations such as FLT3, 
NPM1, and CEBPA confer prognostic significance in adults with normal karyotype AML and help develop a strategy. Patients with mutations in NPM1 and double mutants for CEBPA without FLT3-ITD mutations have a better prognosis and are usually treated with chemotherapy alone. In contrast, patients with FLT3-ITD mutations have an adverse prognosis, as mentioned earlier, and allogeneic transplantation is included in the treatment plan if a donor is available. ${ }^{32}$

\section{FLT3 inhibitors in AML}

Several oral FLT3 tyrosine kinase inhibitors, including lestaurtinib (CEP701), sunitinib malate, sorafenib, and midostaurin (PKC412), have been developed and tested in clinical trials with AML patients. Although there is evidence of antileukemic activity in Phase II studies, most responses are incomplete and transient. ${ }^{33,34}$ Lestaurtinib and midostaurin can safely be combined with standard chemotherapy and are currently tested in clinical trials that compare outcomes when chemotherapy is used with or without those agents. In a randomized trial of therapy for FLT3 mutant AML in first relapse, 224 patients received chemotherapy alone or followed by $80 \mathrm{mg}$ of the FLT3 inhibitor lestaurtinib twice daily. However, lestaurtinib treatment after chemotherapy neither increased response rates nor prolonged survival of patients with FLT3 mutant AML patients. An increase in FLT3 ligand after the administration of induction chemotherapy may impair the ability of these agents to inhibit their target. ${ }^{35}$

\section{Lymphomas}

Non-Hodgkin's lymphoma consists of a diverse group of hematologic malignancies variously derived from B cell progenitors, T cell progenitors, mature B cells, mature T cells, or (rarely) natural killer cells. Hodgkin's lymphoma (HL) is a group of malignant neoplasms characterized by ReedSternberg cells in a reactive cellular background. Although certain gene expression profiles can further subclassify DLBCL and several genetic and molecular abnormalities have been described in lymphoma patients, these are still not used to guide treatment, but this is expected to change soon. ${ }^{36,37}$ Regarding JAK-STAT signaling, it has been shown that this pathway is aberrantly activated in many lymphomas by multiple mechanisms, although only JAK3-activating mutations have been identified. .,38,39 $^{9}$

\section{JAK inhibitors in lymphomas}

Because the JAK-STAT pathway activation has been implicated in the pathogenesis of lymphomas, it became an appealing target for pathway-directed lymphoma therapy.
Nonselective JAK-STAT inhibitors such as AG490 were shown to induce cell death and apoptosis in HL, multiple myeloma, and non-Hodgkin's lymphoma cell lines, ${ }^{40}$ whereas AUH-6-96 was shown to selectively affect cell viability of cancer cells harboring aberrant JAK-STAT signaling by down-regulating the expression of STAT3 downstream target antiapoptotic genes, such as Bcl-xL, and inducing programmed cell death. ${ }^{41}$ Lestaurtinib, an orally bioavailable multikinase inhibitor that has recently been shown to inhibit JAK2 in myeloproliferative disorders, has also been shown to inhibit proliferation and induce apoptosis in HL. ${ }^{42,43}$ AZD1480 showed antiproliferative activity in preclinical models of HL by decreasing the secretion of various cytokines and the expression of the immunosuppressive PD-L1 and PD-L2. Taken together, these preclinical observations provide a rationale to further exploit the potential of JAK inhibitors as antilymphoma agents. ${ }^{44}$

\section{The clinical development of pacritinib}

Pacritinib (SB1518) is a novel, low-molecular weight, selective inhibitor of JAK2 and FLT3 with potent therapeutic application in MPNs, AML, and lymphomas (Figure 1). ${ }^{45}$ The structure and the pharmacological profile of pacritinib were first described by Hart et al, ${ }^{45}$ who subsequently tested it against more than 50 other protein kinases covering all major families of the human protein kinome. ${ }^{45,46}$ It was designed with the aid of computational chemistry as a small-molecule adenosine triphosphate-site competitive inhibitor of JAK2 kinase. It contains a pyrimidine core within a macrocyclic structure and has favorable physicochemical and pharmaceutical properties. It exhibits much higher selectivity for JAK2 than JAK3 or JAK1 (half maximal inhibitory concentration $\left[\mathrm{IC}_{50}\right]=23 \mathrm{nM}, 520 \mathrm{nM}$, and 1,280 nM, respectively). It also inhibits FLT3 and FLT3D835Y mutant, rendering it active not only in MPNs but also in AML. Pacritinib is 3.66-fold more selective for FLT3D835Y mutant $\left(\mathrm{IC}_{50}=6 \mathrm{nM}\right)$ than FLT3 $\left(\mathrm{IC}_{50}=22 \mathrm{nM}\right)$. It inhibits both wild-type JAK2 and the mutant form of JAK2 (JAK2V617F) and has potent antiproliferative activity even in cells without the JAK2V617F mutation. As reviewed later, pacritinib induces lower myelosuppression compared with other JAK inhibitors ${ }^{47,48}$ and is more selective than other FLT3 inhibitors, ${ }^{26}$ whereas the concurrent inhibition of both FLT3 and JAK2 may help override the chemoresistance that results from the interactions between stromal cells and leukemic cells often observed in AML, ${ }^{49}$ making it a very promising anticancer agent. It showed activity against JAK2- and FLT3-dependent leukemic cell 
lines, ${ }^{45}$ and on the basis of its good oral bioavailability, it was selected for evaluation in two mouse tumor models: the $\mathrm{Ba} / \mathrm{F} 3-\mathrm{JAK} 2^{\mathrm{V} 617 \mathrm{~F}}$ and the MV4-11 allograft and xenograft studies, representing cell lines dependent on mutant and FLT3 signaling, respectively. In the JAK2V617F-driven disease model, pacritinib demonstrated dose-dependent tumor growth inhibition ${ }^{45}$ and normalization of splenomegaly and hepatomegaly at well-tolerated doses. In the model of FLT3ITD-driven leukemia, pacritinib demonstrated significant survival benefits, again at very well-tolerated doses. ${ }^{50}$ In other preclinical models of AML, pacritinib in combination with pracinostat, a histone deacetylase inhibitor, showed synergy in tumor growth and reduction in metastasis, whereas a JAK2 or FLT3 signaling decrease was noted, along with normalization of several plasma cytokines, growth factors, and chemokines, providing a rationale for the combination of these two agents in AML patients with JAK2 or FLT3 mutations. ${ }^{51}$

\section{Pharmacokinetics and pharmacodynamics}

The pharmacokinetics (PK) of pacritinib were originally analyzed via oral and intravenous administration in animals (mice, rats, and dogs). In mice, the levels of the drug were higher in the lungs and lower in the brain. Pacritinib is more than $99 \%$ bound to plasma proteins in dog, mouse, and human plasma, as indicated by plasma protein binding studies. As showed by a radioactivity distribution study executed in mice, the highest concentrations of pacritinib were observed in the gastrointestinal system and the bile, whereas the lowest were seen in the central nervous system and bones. ${ }^{52}$

As far as humans are concerned, the oral pharmacokinetics of pacritinib were assessed in three clinical trials (SB15182007-001, SB1518-2007-002, and SB1518-2008-003), with the assessments being made on days 1 and 15 of each cycle. Pacritinib was given every 25-28 days at doses ranging from 100-600 mg. Pacritinib seems to exhibit dose-related, but not dose-proportional, increases in exposure..$^{53,54}$ Two clinical trials in healthy volunteers examined the PK parameters of pacritinib. One of them concerned fasting volunteers $(n=24)$ to whom single doses of the drug $(100,200$, and $400 \mathrm{mg})$ were administered within 4 hours of fasting after dosing and a 14-day washout period between treatments. The median time to reach the peak plasma concentration of the drug after administration $\left(\mathrm{t}_{\max }\right)$ and elimination half life $\left(\mathrm{t}_{1 / 2}\right)$ did not show apparent changes with dose changes. ${ }^{52}$ The second study examined the effect of food on the bioavailability and PK of the drug. Eighteen volunteers received $200 \mathrm{mg}$ of pacritinib after having consumed a high-fat meal and being fasted. Under fed conditions, the mean concentrations, the peak plasma concentration of the drug after administration $\left(\mathrm{C}_{\max }\right)$ and area under the curve were higher than under fasted conditions. ${ }^{52}$

\section{Safety}

The adverse effects (AEs) of pacritinib were evaluated in 30-day repeated oral dose toxicity studies in both mice and dogs. From these studies, the maximum tolerated dose (MTD) of $80 \mathrm{mg}$ / $\mathrm{kg}$ /day was used for calculation of the starting dose in human clinical trials. In the above-mentioned clinical trials involving volunteers, 24 human subjects were treated in a randomized, crossover trial designed to evaluate the $\mathrm{PK}$ of pacritinib at doses of 100, 200, and $400 \mathrm{mg}$. Pacritinib was very well tolerated at the lower doses (100 and $200 \mathrm{mg}$ ), whereas at the dose of 400 $\mathrm{mg}$, AEs noted were mainly gastrointestinal, such as diarrhea, nausea, and vomiting. ${ }^{52}$ In the SB1518-2010-006 study, which assessed the effect of food on the bioavailability and PK of a standard dose of $200 \mathrm{mg}$ of pacritinib, the drug was again well tolerated, with no SAEs reported and no AE leading to discontinuation. Three of 18 people experienced AEs (nausea, headache) that were mild to moderate in intensity. No significant differences under fasted and fed conditions were observed with regard to the safety profile of the drug. ${ }^{52}$

To date, 191 patients with hematological disorders have received pacritinib: 187 of them (97.9\%) experienced treatment-emergent AEs (including both drug-related and drug-unrelated). Grade 3/4 AEs were reported in 123 (64.4\%). The main AEs were gastrointestinal disorders of mild to moderate intensity, with diarrhea, nausea, and vomiting being the most frequent ones. Other reported gastrointestinal disorders were constipation and abdominal pain or distention. ${ }^{52}$

Pacritinib seems to provoke minimal myelosuppression, and hematologic AEs were rare. Of all patients who participated in clinical trials, $19.4 \%$ experienced thrombocytopenia as a treatment-related $\mathrm{AE}$ and a $25.6 \%$ reported anemia as a treatment-emergent AE. Even in patients with very low platelet counts at baseline $(<50,000 / \mu \mathrm{L})$, no dose reductions were required. Other AEs reported were fatigue, peripheral edema, pyrexia, pruritus, dyspnea, cough, anorexia, dizziness, headache, and insomnia.

\section{Clinical trials}

To date, there have been eight clinical trials concerning pacritinib, five of which have been completed, one of which was terminated, and two of which are recruiting (Table 1). ${ }^{52}$

One of the first clinical trials to be launched was an openlabel Phase I/II trial (SB1518-2007-001, clinicaltrials.gov identifier NCT00719836) to establish the MTD of pacritinib 


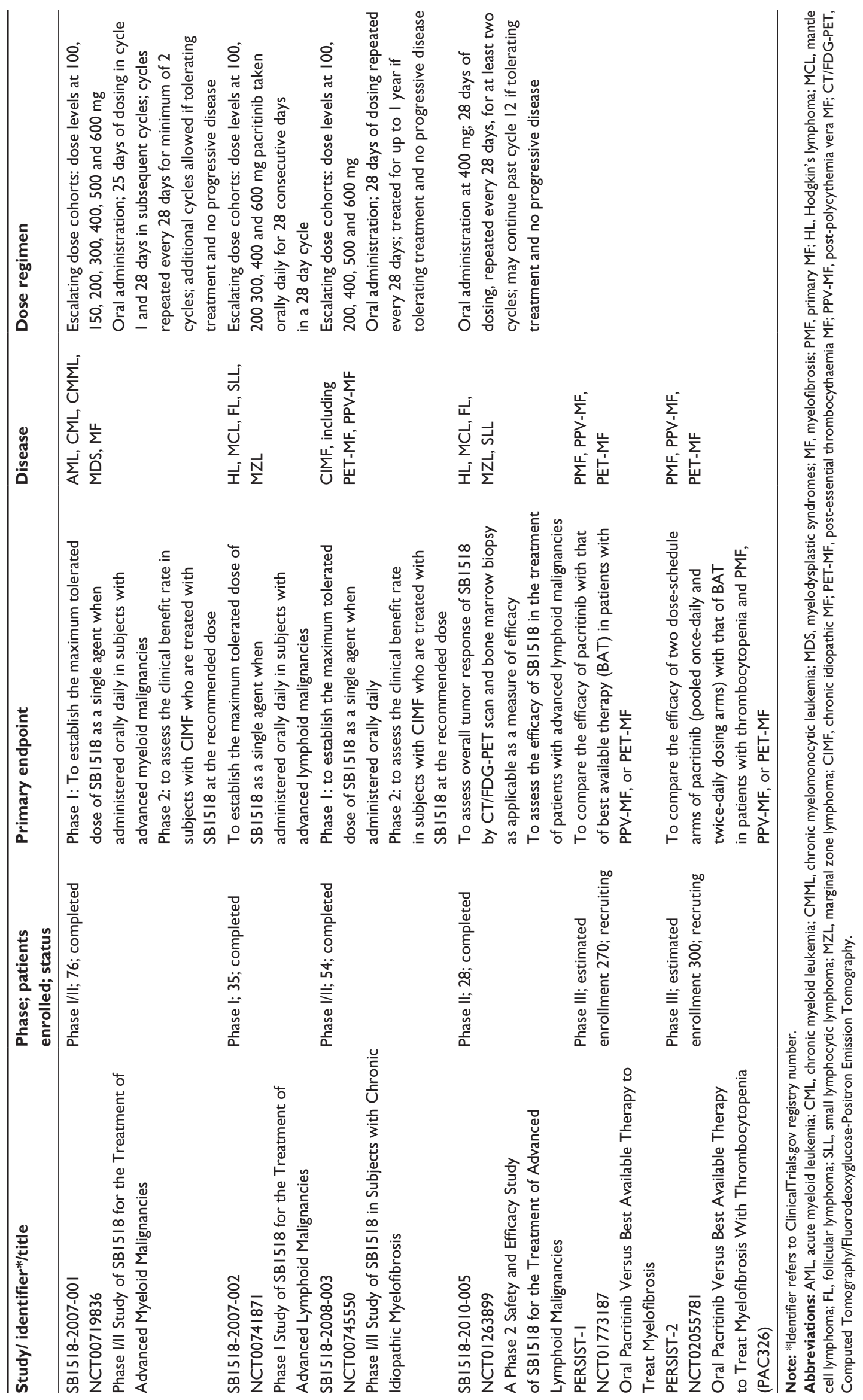


as single agent when administered orally daily in patients with advanced myeloid malignancies and to further assess the clinical benefit rate in patients with MF who were scheduled to be treated at the recommended dose. The total number of patients enrolled was 76, but the first results announced concerned 36 patients (31/36 MF, 5/36 AML) who were treated at 6 dose levels, from 100-600 mg daily, with a median number of prior therapies of two. At $600 \mathrm{mg}$, three of six patients experienced gastrointestinal dose-limiting toxicity. All three recovered and restarted pacritinib at a reduced dose. The most common related AEs recorded were diarrhea $(33 \% ; 4 \%$ grade 3$)$, nausea ( $13 \%$, all grade $1 / 2)$, and thrombocytopenia (4\%, all grade $3 / 4)$. At the time of analysis, $21 \mathrm{MF}$ patients were evaluable for response: seven of 17 (41\%) with palpable splenomegaly had a decrease in spleen size by physical exam of $35 \%$ or more, of whom four $(24 \%)$ had a decrease of $50 \%$ or more. ${ }^{53}$ It was later reported that eight patients achieved a $50 \%$ or higher spleen dimension reduction, and nine patients had a normalization of the number of white blood cells during treatment (two-thirds of whom received doses of $400 \mathrm{mg}$ or higher). ${ }^{52}$

Another open-label Phase I/II study evaluated pacritinib in patients with chronic idiopathic MF (SB1518-2008-003, clinicaltrials.gov identifier CT00745550); 55 patients were enrolled in this study. ${ }^{52,55}$ The purpose of the Phase I part was to establish the MTD of pacritinib when administered orally daily. Five escalating dose cohorts were formed (dose levels $100,200,400,500$, and $600 \mathrm{mg}$ ), and patients received the drug in 28-day cycles repeated every 28 days for up to 1 year if treatments were tolerated and there was no progressive disease. The purpose of the Phase II part of the trial was to assess the clinical benefit rate in subjects treated with pacritinib at the recommended dose (400 mg). In the Phase I part (20 patients), three of the patients had improvement in splenomegaly. The response was estimated according to the MF-international working group criteria, the treatment effect on splenomegaly was measured using magnetic resonance imaging, as in the previous study, and the symptom relief was estimated using the Myelofibrosis Symptom Assessment Form. In the Phase I portion of the study, 20 patients with MF were treated and three $(15 \%)$ achieved clinical improvement in splenomegaly response, whereas one $(5 \%)$ had a partial response. Of note, in three of 14 patients with palpable spleens $5 \mathrm{~cm}$ or more below the left costal margin at baseline, the spleen became nonpalpable on study and responses observed were regardless of the presence of the JAK2V617F mutation.

Magnetic resonance imaging results through week 24 showed that $29(59 \%)$ of 49 evaluable patients had a $25 \%$ or greater reduction in spleen volume, and $27 \%$ of the above 29 patients had a $35 \%$ or greater reduction in spleen volume. Regarding symptom relief, at week 12, all symptoms analyzed (such as abdominal pain, bone pain, early satiety, inactivity, night sweats, and pruritus) were relieved with the exception of fatigue, which was recorded as worsening. However, at week 24, even greater score improvement was observed for all symptoms, and similar improvement was observed in patients with baseline platelet counts lower than $100,000 / \mu \mathrm{L} .^{52}$

In another open-label, Phase I study (SB1518-2007-002, clinicaltrials.gov identifier NCT00741871), 35 patients with relapsed/refractory lymphoid malignancies participated. One enrolled patient withdrew before receiving the drug. The purpose of the study was to establish the MTD, the safety and tolerability, and the PK/pharmacodynamic profile of pacritinib. Fifteen of the 35 patients had classic HL, 10 had FL, 5 had MCL, 4 had DLBCL, and 1 had SLL. Patient cohorts received escalating doses of pacritinib orally once daily for 28-day cycles. The starting dose was $100 \mathrm{mg}$, and dose escalation was guided by the safety evaluation during the first cycle of treatment. Response was evaluated after 8 weeks. The MTD was not reached; however, $400 \mathrm{mg} /$ day was the dose recommended for the second part of the study, as suggested by the PK/pharmacodynamic data obtained. ${ }^{54}$ Responses in 31 patients with at least a single follow-up tumor assessment were as follows: four patients had partial remission (two patients with MCL at doses of 300 and $400 \mathrm{mg} /$ day and two with FL at doses of 400 and $600 \mathrm{mg} /$ day), and 17 had stable disease (seven with FL, seven with HL, two with MCL, and one with SLL), but 13 of the 17 patients with stable disease had tumor mass reductions of $4 \%-45 \%$. None of the DLBCL patients responded, but the sample size was too small to draw conclusions. ${ }^{52,54}$ The most frequent events ( $>10 \%$ of patients) were gastrointestinal toxicities: diarrhea (32\%), nausea/ vomiting (29\%), constipation (26\%), and decreased appetite $(12 \%)$. Most of the serious adverse events were unrelated to the study drug. Of note, in lymphoma patients, in contrast to MPN patients, the investigators could not find any useful biomarker of activity, as the baseline plasma level of cytokines and chemokines did not correlate with response. ${ }^{54,56}$

Another open-label, Phase II clinical trial (SB1518-2010005, clinicaltrials.gov identifier NCT01263899) studied the efficacy of pacritinib in the treatment of patients with advanced lymphoid malignancies including HL, MCL, and indolent lymphoma (FL, lymphoplasmacytic lymphoma, marginal zone lymphoma, and SLL). Twenty-eight patients were enrolled (ten with HL, ten with indolent lymphoma, and eight with MCL). The purpose of the trial was to assess the overall tumor response of pacritinib by CT/18-fluoro-deoxyglucose 
positron emission tomography scan and bone marrow biopsy, the durability of response, and the safety/tolerability of the drug. The patients received $400 \mathrm{mg}$ per day orally for at least two 28-day cycles repeated every 28 days. Response was assessed using the international working group Revised Response Criteria for Malignant Lymphoma. ${ }^{57}$ Of 24 patients with at least a single follow-up assessment, 17 patients had stable disease (five patients with HL, four with MCL, and eight with FL), one patient with FL had partial remission, and one with HL achieved a complete remission. ${ }^{52}$

A Phase II trial (NCT01436084), which enrolled eight adult patients with myelodysplastic syndrome (low, intermediate 1 , intermediate 2 , and high risk) who had received at least a single line of prior therapy, has been terminated based on collaborating sponsor decision.

At this time, a Phase III clinical trial (Oral Pacritinib Versus Best Available Therapy to Treat Myelofibrosis, clinicaltrials. gov identifier NCT01773187) is still recruiting to compare the efficacy of pacritinib with that of best available therapy (any treatment except for tyrosine kinase inhibitors) in patients with PMF, PPV-MF, or PET-MF, whereas another one (Oral Pacritinib Versus Best Available Therapy to Treat Myelofibrosis With Thrombocytopenia, clinicaltrials.gov identifier NCT02055781) will compare the efficacy of two dose-schedule groups of pacritinib (pooled once-daily and twice-daily dosing groups) with that of best available therapy in patients with PMF PPV-MF, PET-MF, and thrombocytopenia (Table 1).

\section{Conclusion}

Pacritinib (previously known as SB-1518) is an innovative selective inhibitor of JAK2 and FLT3 providing potential in the treatment of hematological malignancies. Pacritinib has potent antiproliferative activity in JAK2 and/or FLT3 activitydependent cell lines and the ability to promote apoptosis and inhibit the STAT pathway. It has showed antitumor activity in mouse models bearing the JAK2 and FLT3 mutations, and clinical trials further support its efficacy and safety profile. In MF patients, pacritinib led to disease responses, with improvement in splenomegaly and constitutional symptom relief. In patients with various lymphoma subtypes, clinical benefit was also observed. Pacritinib induces lower myelosuppression compared with other JAK inhibitors, ${ }^{47,48}$ and no dose reductions for thrombocytopenia were necessary in Phase II trials. The most common treatment-related adverse events recorded were gastrointestinal toxicity. Pacritinib is also more selective than other FLT3 inhibitors, ${ }^{26}$ whereas the concurrent inhibition of both FLT3 and JAK2 may help override the chemoresistance resulting from the interactions between stromal cells and leukemic cells often observed in AML, ${ }^{49}$ making it a very promising anticancer agent. Although the development of pacritinib continues with ongoing Phase III trials, it remains to be determined whether it can considerably affect disease progression or alter bone marrow histologic features (eg, fibrosis) in MPNs, and whether it can alter the prognosis of FLT3-ITD mutant AML. As we move from molecule-targeted to pathway-directed therapy, it will be interesting to see future clinical development focusing on optimal combination agents that target multiple mechanisms leading hopefully to deeper and sustainable responses improving the survival of our patients.

\section{Disclosure}

The authors report no conflicts of interest in this work.

\section{References}

1. Harrison DA. The Jak/STAT pathway. Cold Spring Harb Perspect Biol. 2012;4(3):a011205.

2. Furqan M, Akinleye A, Mukhi N, Mittal V, Chen Y, Liu D. STAT inhibitors for cancer therapy. J Hematol Oncol. 2013;6(1):90.

3. Baxter EJ, Scott LM, Campbell PJ, et al; Cancer Genome Project. Acquired mutation of the tyrosine kinase JAK2 in human myeloproliferative disorders. Lancet. 2005;365(9464):1054-1061.

4. James C, Ugo V, Le Couédic JP, et al. A unique clonal JAK2 mutation leading to constitutive signalling causes polycythaemia vera. Nature. 2005;434(7037):1144-1148.

5. Steensma DP, Dewald GW, Lasho TL, et al. The JAK2 V617F activating tyrosine kinase mutation is an infrequent event in both "atypical" myeloproliferative disorders and myelodysplastic syndromes. Blood. 2005;106(4):1207-1209.

6. Scott LM, Campbell PJ, Baxter EJ, et al. The V617F JAK2 mutation is uncommon in cancers and in myeloid malignancies other than the classic myeloproliferative disorders. Blood. 2005;106(8):2920-2921.

7. Nangalia J, Massie CE, Baxter EJ, et al. Somatic CALR mutations in myeloproliferative neoplasms with nonmutated JAK2. $N$ Engl J Med. 2013;369(25):2391-2405.

8. Klampfl T, Gisslinger H, Harutyunyan AS, et al. Somatic mutations of calreticulin in myeloproliferative neoplasms. $N$ Engl $J$ Med. 2013;369(25):2379-2390.

9. Ding BB, Yu JJ, Yu RY, et al. Constitutively activated STAT3 promotes cell proliferation and survival in the activated B-cell subtype of diffuse large B-cell lymphomas. Blood. 2008;111(3):1515-1523.

10. Green MR, Monti S, Rodig SJ, et al. Integrative analysis reveals selective 9p24.1 amplification, increased PD-1 ligand expression, and further induction via JAK2 in nodular sclerosing Hodgkin lymphoma and primary mediastinal large B-cell lymphoma. Blood. 2010;116(17): 3268-3277.

11. Rosenwald A, Wright G, Leroy K, et al. Molecular diagnosis of primary mediastinal B cell lymphoma identifies a clinically favorable subgroup of diffuse large B cell lymphoma related to Hodgkin lymphoma. J Exp Med. 2003;198(6):851-862.

12. Joos S, Küpper $\mathrm{M}$, Ohl S, et al. Genomic imbalances including amplification of the tyrosine kinase gene JAK2 in CD30+ Hodgkin cells. Cancer Res. 2000;60(3):549-552.

13. Melzner I, Bucur AJ, Brüderlein S, et al. Biallelic mutation of SOCS-1 impairs JAK2 degradation and sustains phospho-JAK2 action in the MedB-1 mediastinal lymphoma line. Blood. 2005;105(6):2535-2542.

14. Weniger MA, Melzner I, Menz CK, et al. Mutations of the tumor suppressor gene SOCS-1 in classical Hodgkin lymphoma are frequent and associated with nuclear phospho-STAT5 accumulation. Oncogene. 2006;25(18):2679-2684. 
15. Van Roosbroeck K, Cox L, Tousseyn T, et al. JAK2 rearrangements, including the novel SEC31A-JAK2 fusion, are recurrent in classical Hodgkin lymphoma. Blood. 2011;117(15):4056-4064.

16. Gupta M, Han JJ, Stenson M, et al. Elevated serum IL-10 levels in diffuse large B-cell lymphoma: a mechanism of aberrant JAK2 activation. Blood. 2012;119(12):2844-2853.

17. Aldinucci D, Gloghini A, Pinto A, De Filippi R, Carbone A. The classical Hodgkin's lymphoma microenvironment and its role in promoting tumour growth and immune escape. J Pathol. 2010;221(3):248-263.

18. Lee JW, Kim YG, Soung YH, et al. The JAK2 V617F mutation in de novo acute myelogenous leukemias. Oncogene. 2006;25(9):1434-1436.

19. Döhner K, Du J, Corbacioglu A, Scholl C, Schlenk RF, Döhner H. JAK2V617F mutations as cooperative genetic lesions in $\mathrm{t}(8 ; 21)$-positive acute myeloid leukemia. Haematologica. 2006;91(11):1569-1570.

20. Ikezoe T, Kojima S, Furihata M, et al. Expression of p-JAK2 predicts clinical outcome and is a potential molecular target of acute myelogenous leukemia. Int J Cancer. 2011;129(10):2512-2521.

21. Hatzimichael E, Georgiou G, Benetatos L, Briasoulis E. Gene mutations and molecularly targeted therapies in acute myeloid leukemia. $\mathrm{Am} \mathrm{J}$ Blood Res. 2013;3(1):29-51.

22. Steensma DP, McClure RF, Karp JE, et al. JAK2 V617F is a rare finding in de novo acute myeloid leukemia, but STAT3 activation is common and remains unexplained. Leukemia. 2006;20(6):971-978.

23. Rosnet O, Schiff C, Pébusque MJ, et al. Human FLT3/FLK2 gene: cDNA cloning and expression in hematopoietic cells. Blood. 1993;82(4):1110-1119.

24. deLapeyrière O, Naquet P, Planche J, et al. Expression of Flt3 tyrosine kinase receptor gene in mouse hematopoietic and nervous tissues. Differentiation. 1995;58(5):351-359.

25. Zhang S, Mantel C, Broxmeyer HE. Flt3 signaling involves tyrosylphosphorylation of SHP-2 and SHIP and their association with Grb2 and Shc in Baf3/Flt3 cells. J Leukoc Biol. 1999;65(3):372-380.

26. Hart S, Goh KC, Novotny-Diermayr V, et al. Pacritinib (SB1518), a JAK2/FLT3 inhibitor for the treatment of acute myeloid leukemia. Blood Cancer J. 2011;1(11):e44.

27. Cervantes F, Dupriez B, Pereira A, et al. New prognostic scoring system for primary myelofibrosis based on a study of the International Working Group for Myelofibrosis Research and Treatment. Blood. 2009;113(13):2895-2901.

28. Guardiola P, Anderson JE, Bandini G, et al. Allogeneic stem cell transplantation for agnogenic myeloid metaplasia: a European Group for Blood and Marrow Transplantation, Société Française de Greffe de Moelle, Gruppo Italiano per il Trapianto del Midollo Osseo, and Fred Hutchinson Cancer Research Center Collaborative Study. Blood. 1999;93(9):2831-2838.

29. Cervantes F, Alvarez-Larrán A, Domingo A, Arellano-Rodrigo E, Montserrat E. Efficacy and tolerability of danazol as a treatment for the anaemia of myelofibrosis with myeloid metaplasia: long-term results in 30 patients. Br J Haematol. 2005;129(6):771-775.

30. Mesa RA, Yao X, Cripe LD, et al. Lenalidomide and prednisone for myelofibrosis: Eastern Cooperative Oncology Group (ECOG) phase 2 trial E4903. Blood. 2010;116(22):4436-4438.

31. Verstovsek S, Mesa RA, Gotlib J, et al. A double-blind, placebocontrolled trial of ruxolitinib for myelofibrosis. $N$ Engl $J$ Med 2012;366(9):799-807.

32. Döhner H, Estey EH, Amadori S, et al; European LeukemiaNet. Diagnosis and management of acute myeloid leukemia in adults: recommendations from an international expert panel, on behalf of the European LeukemiaNet. Blood. 2010;115(3):453-474.

33. Fischer T, Stone RM, Deangelo DJ, et al. Phase IIB trial of oral Midostaurin (PKC412), the FMS-like tyrosine kinase 3 receptor (FLT3) and multi-targeted kinase inhibitor, in patients with acute myeloid leukemia and high-risk myelodysplastic syndrome with either wild-type or mutated FLT3. J Clin Oncol. 2010;28(28):4339-4345.

34. Knapper S, Burnett AK, Littlewood T, et al. A phase 2 trial of the FLT3 inhibitor lestaurtinib (CEP701) as first-line treatment for older patients with acute myeloid leukemia not considered fit for intensive chemotherapy. Blood. 2006;108(10):3262-3270.
35. Levis M, Ravandi F, Wang ES, et al. Results from a randomized trial of salvage chemotherapy followed by lestaurtinib for patients with FLT3 mutant AML in first relapse. Blood. 2011;117(12):3294-3301.

36. Ghielmini M, Vitolo U, Kimby E, et al; Panel Members of the 1st ESMO Consensus Conference on Malignant Lymphoma. ESMO Guidelines consensus conference on malignant lymphoma 2011 part 1: diffuse large B-cell lymphoma (DLBCL), follicular lymphoma (FL) and chronic lymphocytic leukemia (CLL). Ann Oncol. 2013;24(3):561-576.

37. Dreyling M, Thieblemont C, Gallamini A, et al. ESMO Consensus conferences: guidelines on malignant lymphoma. part 2: marginal zone lymphoma, mantle cell lymphoma, peripheral T-cell lymphoma. Ann Oncol. 2013;24(4):857-877.

38. Elliott NE, Cleveland SM, Grann V, Janik J, Waldmann TA, Davé UP. FERM domain mutations induce gain of function in JAK3 in adult T-cell leukemia/lymphoma. Blood. 2011;118(14):3911-3921.

39. Koskela HL, Eldfors S, Ellonen P, et al. Somatic STAT3 mutations in large granular lymphocytic leukemia. $N$ Engl J Med. 2012;366(20): 1905-1913.

40. Alas S, Bonavida B. Inhibition of constitutive STAT3 activity sensitizes resistant non-Hodgkin's lymphoma and multiple myeloma to chemotherapeutic drug-mediated apoptosis. Clin Cancer Res. 2003;9(1): 316-326.

41. Kim BH, Yin $\mathrm{CH}$, Guo Q, et al. A small-molecule compound identified through a cell-based screening inhibits JAK/STAT pathway signaling in human cancer cells. Mol Cancer Ther. 2008;7(9): 2672-2680.

42. Diaz T, Navarro A, Ferrer G, et al. Lestaurtinib inhibition of the Jak/ STAT signaling pathway in hodgkin lymphoma inhibits proliferation and induces apoptosis. PLoS ONE. 2011;6(4):e18856.

43. Hexner EO, Serdikoff C, Jan M, et al. Lestaurtinib (CEP701) is a JAK2 inhibitor that suppresses JAK2/STAT5 signaling and the proliferation of primary erythroid cells from patients with myeloproliferative disorders. Blood. 2008;111(12):5663-5671.

44. Derenzini E, Lemoine M, Buglio D, et al. The JAK inhibitor AZD1480 regulates proliferation and immunity in Hodgkin lymphoma. Blood Cancer J. 2011;1(12):e46.

45. Hart S, Goh KC, Novotny-Diermayr V, et al. SB1518, a novel macrocyclic pyrimidine-based JAK2 inhibitor for the treatment of myeloid and lymphoid malignancies. Leukemia. 2011;25(11):1751-1759.

46. Goh KC, Ong WC, Hu C, et al. SB1518: a potent and orally active JAK2 inhibitor for the treatment of myeloproliferative disorders. Blood. 2007;110(Abstract):538.

47. Verstovsek S, Kantarjian H, Mesa RA, et al. Safety and efficacy of INCB018424, a JAK1 and JAK2 inhibitor, in myelofibrosis. $N$ Engl $J$ Med. 2010;363(12):1117-1127.

48. Santos FP, Kantarjian HM, Jain N, et al. Phase 2 study of CEP-701, an orally available JAK2 inhibitor, in patients with primary or postpolycythemia vera/essential thrombocythemia myelofibrosis. Blood. 2010;115(6):1131-1136.

49. Weisberg E, Liu Q, Nelson E, et al. Using combination therapy to override stromal-mediated chemoresistance in mutant FLT3-positive AML: synergism between FLT3 inhibitors, dasatinib/multi-targeted inhibitors and JAK inhibitors. Leukemia. 2012;26(10):2233-2244.

50. William AD, Lee AC, Blanchard S, et al. Discovery of the macrocycle 11-(2-pyrrolidin-1-yl-ethoxy)-14,19-dioxa-5,7,26-triazatetracyclo[19.3.1.1(2,6).1(8,12)] heptacosa-1(25),2(26),3,5,8,10,12(27), 16,21,23-decaene (SB1518), a potent Janus kinase 2/fms-like tyrosine kinase-3 (JAK2/FLT3) inhibitor for the treatment of myelofibrosis and lymphoma. J Med Chem. 2011;54(13):4638-4658.

51. Novotny-Diermayr V, Hart S, Goh KC, et al. The oral HDAC inhibitor pracinostat (SB939) is efficacious and synergistic with the JAK2 inhibitor pacritinib (SB1518) in preclinical models of AML. Blood Cancer J. 2012;2(5):e69.

52. Verstovsek S, Machida C, Dean JP, Myint H. Pacritinib. Inhibitor of tyrosine-protein kinase JAK2, inhibitor of FLT-3, treatment of myelofibrosis. Drugs Future. 2013;38(6):375. 
53. Verstovsek S, Odenike O, Scott B, et al. Phase I dose-escalation trial of SB1518, a novel JAK2/FLT3 inhibitor, in acute and chronic myeloid diseases, including primary or post-essential thrombocythemia/polycythemia vera myelofibrosis. Blood. 2009;114(Abstract):3905.

54. Younes A, Romaguera J, Fanale M, et al. Phase I study of a novel oral Janus kinase 2 inhibitor, SB1518, in patients with relapsed lymphoma: evidence of clinical and biologic activity in multiple lymphoma subtypes. J Clin Oncol. 2012;30(33):4161-4167.
55. Verstovsek S, Deeg HJ, Odenike O, et al. Phase $1 / 2$ study of SB1518, a novel JAK2/FLT3 inhibitor, in the treatment of primary myelofibrosis. Blood. 2010;116(Abstract):3082.

56. Derenzini E, Younes A. Targeting the JAK-STAT pathway in lymphoma: a focus on pacritinib. Expert Opin Investig Drugs. 2013;22(6):775-785.

57. Cheson BD, Pfistner B, Juweid ME, et al; International Harmonization Project on Lymphoma. Revised response criteria for malignant lymphoma. J Clin Oncol. 2007;25(5):579-586.

Journal of Blood Medicine

\section{Publish your work in this journal}

The Journal of Blood Medicine is an international, peer-reviewed, open access, online journal publishing laboratory, experimental and clinical aspects of all topics pertaining to blood based medicine including but not limited to: Transfusion Medicine; Blood collection, Donor issues, Transmittable diseases, and Blood banking logistics; Immunohematology; Artificial and alternative

\section{Dovepress}

blood based therapeutics; Hematology; Biotechnology/nanotechnology of blood related medicine; Legal aspects of blood medicine; Historical perspectives. The manuscript management system is completely online and includes a very quick and fair peer-review system. Visit http://www.dovepress.com/ testimonials.php to read real quotes from published authors.

Submit your manuscript here: http://www.dovepress.com/Journal-of-blood-medicine-journal 